\title{
EDITORIAL
}

\section{Positron Emission Tomography in Mesothelioma Patients}

\author{
Gaia Grassetto, Domenico Rubello
}

Department of Nuclear Medicine, PET Center, 'Santa Maria della Misericordia' Hospital, Viale Tre Martiri 140, 45100 Rovigo, Italy

$I^{2}$ n the study by Mavi and co-workers from Dr. Alavi's group, published in the present issue of Molecular Imaging \& Biology [1], the authors reported their experience with the $18 \mathrm{~F}$-fluorodeoxyglucose positron emission tomography (FDG-PET) in malignant pleural mesothelioma patients (MPM). In particular, they have focused their attention on the diagnostic phase of this disease that is still a challenge in all its aspects: diagnosis, staging and therapy [2]. Above all, it is worth noting that mesothelioma is a challenging disease to image with any modality, and no single imaging approach captures the information necessary to direct all aspects of patient's management [3]. The clinical presentation of mesothelioma, especially if in the early phase, is insidious and not specific [4]. Dyspnoea is the predominant symptom, in general secondary to tumour effusion, and only when the disease becomes advanced that chest pain related to tumour infiltration of chest wall and intercostals nerves also appears [4]. Therefore, to make diagnosis of mesothelioma, the physician has to think about it. Moreover, diffuse pleural thickening, a very frequent sign at computed tomography (CT) of patients with asbestos exposure history, is not specific for a malignant process of the pleura [5-8]. Therefore, often, it is necessary to utilise an invasive approach, like thoracoscopy or open biopsy, to get to the correct and definitive diagnosis [5].

As already described in a preliminary combined experimental/clinical research study published in The Journal of Nuclear Medicine by Alavi and co-workers [9], in this new study, the authors further investigated and confirmed the usefulness of FDG-PET acquisition technique based on a dual-time approach for diagnosis of pleural mesothelioma. The authors studied 55 patients, a consistent number of

Invited editorial about the paper by "Mavi A, Basu S, Cermik T, Urhan M, Batali m, Thiruvenkatasamy D, Houseni M, Dadparvar S, Alavi A, entitled "Potential of dual-time point FDG-PET imaging in differentiating malignant from benign pleural disease" in press in Molecular Imaging \& Biology.

Correspondence to: Domenico Rubello; e-mail: domenico.rubello@libero.it people, since the mesothelioma is a rare tumour, and submitted all of them to a dual-time FDG-PET (the first PET scan acquired after $60 \mathrm{~min}$ and the second PET scan acquired after $90 \mathrm{~min}$ from tracer injection). They found that the $\triangle S_{U V}$ max between the two PET scans was very useful to determine if a metabolically active pleural lesion was malignant or not. In fact, they found a statistical significant increase of $\triangle \mathrm{SUV}_{\max }$ in lesions confirmed to be malignant at histology (MPM; new or recurrent disease). On the contrary, this was not true for benign pleural lesions, where $\Delta \mathrm{SUV}_{\max }$ decreased significantly in the authors' experience. The authors recorded only one benign lesion with the increase of $\triangle S U V_{\max }$ (only one false positive result). Thus, it was concluded that this technique is accurate and, with respect on the researches that utilised only one PET acquisition and only one SUV value, is able to increase not only the specificity but also the sensitivity. In fact, small size lesions could be seen only in the second PET study, when they have had concentrated the radioactive glucose.

These clinical data confirm a previous experimental study published by Alavi himself [9] in the abovementioned study in which the research consisted of three steps: the first in vitro, the second in animals and the third in a group of 26 patients. In this preliminary study, the authors found that the SUV of malignant pleural lesions increase over time; conversely, the SUV of inflammatory lesions decrease or remain stable over time.

This new study [1] published in the present issue of Molecular Imaging \& Biology together with the previous preliminary study published in The Journal of Nuclear Medicine [9], at our knowledge, are the unique studies that evaluate the role of the dual-time FDG-PET acquisition technique in the diagnosis of MPM. Some other studies on MPM that were reported in the literature described the value of FDG-PET with the usual technique, which consists on the acquisition of images only at $60 \mathrm{~min}$ after the injection of tracer and with the semi-quantitative analysis based on the evaluation of the unique SUV measurement available: These studies place the cutoff of SUV around the number 2.0/2.5 
$[2,10-12]$ and, to diagnose the mesothelioma, found a sensitivity of more than $90 \%[2,11,13,14]$.

It is of interest to point out that some false negative results were described in the literature in the presence of small-sized malignant pleural lesions characterised by a low tracer uptake as well as in some cases of mesotheliomas of the epithelial subtype because they can not be glucose-avid $[8,11]$. Nevertheless, relatively low specificity values have been reported, around $80 \%[2,11-15]$ mainly related to inflammatory conditions, especially in patients with asbestos exposure history, who develop FDG-avid lesions (pleuritis, benign plaques, parapneumonic effusion, tuberculosis and talc pleurodesis) $[2,8,11-13,16,17]$. These conditions are difficult to distinguish from malignant lesions; in these cases, before recurring to invasive diagnostics methods, it may be helpful to utilise the FDG/PET dual-time protocol proposed by Mavi and co-workers [1].

In conclusion, since the dual-time FDG/PET protocol seems to be useful in providing a differential diagnosis between malignant and benign pleural lesions, thus potentially avoiding invasive diagnostic procedures, it will be necessary to elaborate others researches to confirm the high sensitivity and specificity of this FDG/PET protocol and further investigate the optimal time for the acquisition of the second PET scan. In fact, as observed by the authors of the present study [1], some tumour cells of mesothelioma continue to take up glucose longer than 90 min after tracer injection and require several hours to reach maximum level in FDG uptake. Therefore, if the second PET is acquired at $90 \mathrm{~min}$ from injection, some lesions could be lost.

Moreover, at our knowledge, there is no study concerning the use of dual-time protocol with hybrid PET/CT. Maybe, the fusion between functional and morphological imaging can further increase the sensibility and specificity and accuracy of this procedure. In fact, the CT is used for attenuation correction and allows more precise quantitative analysis of tumour metabolism [6]. At the moment, it is clear that PET/CT is becoming more and more useful for mesothelioma staging because, thanks to the morphological imaging, PET/CT can provide further information about staging " $\mathrm{T}$ " and " $\mathrm{N}$ " and overall about the presence of distant metastasis $[6,8,18]$. Moreover PET/CT seems very useful to define the prognosis (higher is the metabolic activity of a lesion, worse is expect to be the prognosis) and the response to treatment $[2,3,6,12,19]$.

\section{References}

1. Mavi A, Basu S, Cermik T, Urhan M, Batali M, Thiruvenkatasamy D, Houseni M, Dadparvar S, Alavi A: Potential of dual-time point FDGPET imaging in differentiating malignant from benign pleural disease. Mol Imaging Biol (this issue)

2. Gerbaudo VH (2003) 18f-FDG imaging of malignant pleural mesothelioma: scentiam impendere vero.... Nucl Med Common 24:609-614

3. Armato S, Entwisle J, Truong M, Nowak A, Ceresoli GL, Zhao B, Misri R, Kindler H (2008) Current state and future directions of pleural mesothelioma imaging-meeting report. Lung Cancer 99:411420

4. Kent M, Rice D, Flores R (2008) Diagnosis, staging and surgical treatment of malignant pleural mesothelioma. Curr Treat Options Oncol 9:158-170

5. Haberkorn U (2004) Positron emission tomography in the diagnosis of mesothelioma. Lung Cancer 45S:S73-S76

6. Ambrosini V, Rubello D, Nanni C, Farshad M, Castellucci P, Franchi R, Fabbri M, Rampin L, Crepaldi G, Al-Nahhas A, Fanti S (2005) Additional value of hybrid PET/CT fusion imaging vs conventional CT scan alone in the staging and management of patients with malignant pleural mesothelioma. Nucl Med Rev 8(2):111-115

7. Nanni C, Castellucci P, Farsad M, Fanti S (2004) Role of 18F-FDG PET for evaluating malignant pleural mesothelioma. Cancer Biother Radiopharm 19:149-154

8. Yamamuro M, Gerbaudo VH, Gill RR, Jacobson FL, Sugarbaker DJ, Hatabu H (2007) Morphologic and functional imaging of malignant pleural mesothelioma. Eur J Radiol 64:356-366

9. Zhuang H, Pourdehnad M, Lambright E, Yamamoto A, Lanuti M, Li $P$ et al (2001) Dual time point 18F-FDG PET imaging for differentiating malignant from inflammatory processes. J Nucl Med 42:1412-1417

10. Meigna M, Paone G (2006) 18-Fluoro-deoxy-glucose (18FDG) positron emission tomography (PET) for the evaluation of malignant pleural disease. Rev Pneumol Clin 62(2):128-134

11. Benard F, Sterman D, Smith RJ, Kaiser LR, Albelda SM, Alavi A (1998) Metabolic imaging of malignant pleural mesothelioma with fluorodeoxyglucose positron emission tomography. Chest 114:713-722

12. Spitilli MG, Treglia G, Calcagni ML, Giordano A (2007) Malignant pleural mesothelioma: utility of 18F-FDG PET. Ann Ital Chir 78:393-396

13. Schneider DB, Clary-Macy C, Challa S et al (2000) Positron emission tomography in the stging and pre-operative evaluation of malignant pleural mesothelioma. J Thorac Cardiovasc Surg 120:128-133

14. Gerbaudo VH, Sugarbaker DJ, Britz-Cunningham S, Di Carli MF, Mauceri C, Treve ST (2002) Assesment of malignant pleural mesothelioma with $18 \mathrm{~F}$-FDG dual-head gamma-camera coincidence imaging: comparison with histopathology. J Nucl Med 43:1144-1149

15. Kramer H, Pieterman RM, Slebos DJ, Timens W, Vaalburg W, Koeter GH, Groen HJM (2004) PET for evaluation of pleural thickening observed on CT. J Nucl Med 45:995-998

16. Bury T, Paulus P, Dowlati A, Corhay JL, Rigo P, Radermecker MF (1997) Evaluation of pleural disease with FDG-PET imaging: preliminary report. Thorax 52:187-189

17. Bunyaviroch T, Coleman RE (2006) PET evaluation of Lung Cancer. J Nucl Med 47(3):451-469

18. Truong MT, Marom EM, Erasmus JJ (2006) Preoperative evaluation of patients with malignant pleural mesothelioma: role of integrated CTPET imaging. J Thorac Imaging 21(2):146-153

19. Flores RM (2005) The role of PET in the surgical management of malignant pleural mesothelioma. Lung Cancer 49:S27-S32 